\title{
Specific heat of segmented Heisenberg quantum spin chains in $\left(\mathbf{Y b}_{1-x} \mathbf{L u}_{x}\right)_{4} \mathbf{A} s_{3}$
}

\author{
R. Matysiak \\ Institute of Engineering and Computer Education, University of Zielona Góra, \\ ul. prof. Z. Szafrana 4, 65-516 Zielona Góra Poland \\ P. Gegenwart \\ Max Planck Institute for Chemical Physics of Solids, 01187 Dresden, Germany and \\ I. Physikalisches Institut, Georg-August-Universität Göettingen, \\ Friedrich-Hund-Platz 1, 37077 Göettingen, Germany \\ A. Ochiai \\ Center for Low Temperature Science, Tohoku University, Sendai 980-8578, Japan \\ M. Antkowiak, G. Kamieniarz \\ Computational Physics Division, Faculty of Physics, \\ A. Mickiewicz University, ul. Umultowska 85, 61-614 Poznań, Poland \\ F. Steglich \\ Max Planck Institute for Chemical Physics of Solids, 01187 Dresden, Germany
}

(Dated: November 10, 2018)

\begin{abstract}
We report low-temperature specific heat, $C(T)$, measurements on $\left(\mathrm{Yb}_{1-x} \mathrm{Lu}_{x}\right)_{4} \mathrm{As}_{3}$ with $x=$ 0.01 and $x=0.03$, where nonmagnetic Lu atoms are randomly distributed on antiferromagnetic $S=1 / 2$ Heisenberg chains with $J / k_{\mathrm{B}}=28 \mathrm{~K}$. The observed reduction of $C$ below $15 \mathrm{~K}$ with increasing $x$ is accurately described by quantum transfer matrix simulations without any adjustable parameter, implying that the system is an excellent experimental realization of segmented quantum spin chains. Finite-size effects consistent with conformal-field theory predictions are leading to the formation of an effective low-energy gap. The size of the gap increases with Lu content and accounts for the impurity driven reduction of the specific heat. For both concentrations our results verify experimentally the low temperature scaling behavior established theoretically and also confirm the value of $J$ determined from pure $\mathrm{Yb}_{4} \mathrm{As}_{3}$.
\end{abstract}

PACS numbers: 75.10.Jm, 75.40.Cx, 75.40.Mg,71.55.Ak

\section{INTRODUCTION}

Antiferromagnetic (AF) spin chains have attracted a lot of theoretical and experimental interest due to their intrinsic quantum properties. Theoretical description of such simple systems is less complex compared to higher dimensional spin interactions and still their analysis is very useful. The ground state of integer spin chains was predicted disordered with a gap in the excitation spectrum [1]. The presence of the energy gap between the ground state and the lowest-excited states has been realized in real systems [2, 3] and confirmed in simulations [3, 4].

The importance of computer simulations in studies of the low-dimensional quantum spin systems [5, 6] has recently increased as a result of progress in the methods of simulations and increasing computer power [7, 8]. For the spin chains the simulation techniques have brought reliable results of high accuracy, which can be verified

\footnotetext{
*Electronic address: r.matysiak@eti.uz.zgora.pl
}

${ }^{\dagger}$ Electronic address: gjk@amu.edu.pl sometimes by the exact theoretical solutions and can be also used to verify the approximate theoretical results 9, 10]. Among the various methods of computer simulations the quantum transfer matrix (QTM) technique plays an important role. Its accuracy and advantages were presented in a number of publications [11 13].

The theory of the ideally uniform $\mathrm{S}=1 / 2$ AF Heisenberg chain is well established [9]. An energy gap may also exist in these non-integer spin systems as a consequence of the effect of a staggered magnetic field [14]. A typical system displaying such behaviour is $\mathrm{Yb}_{4} \mathrm{As}_{3}$ [15]. At high temperatures, this system displays a cubic metallic mixed valence phase. Upon cooling to below $300 \mathrm{~K}$, it undergoes a charge ordering transition, coupled to a structural distortion, which leads to the formation of domains. In this phase magnetic $\mathrm{Yb}^{3+}$ ions form one-dimensional chains.

Low-temperature properties of $\mathrm{Yb}_{4} \mathrm{As}_{3}$ are described by effective $S=1 / 2$ spin chains with AF interactions and staggered field [10, 16, 17]. Previously, we have used the QTM method and the Bethe ansatz solution [9] to reproduce the low-temperature specific heat of $\mathrm{Yb}_{4} \mathrm{As}_{3}$ at zero field, as well as in the presence of an external field, which induces a gap in the low-energy excitations [18, 19].

In this paper, we investigate the reduction of the spe- 
cific heat and the low-energy excitations in $\mathrm{Yb}_{4} \mathrm{As}_{3}$ with random dilution of the magnetic moments. Since magnetic $\mathrm{Yb}^{3+}$ is chemically identical to non-magnetic $\mathrm{Lu}^{3+}$ and the charge ordering in $\left(\mathrm{Yb}_{1-x} \mathrm{Lu}_{x}\right)_{4} \mathrm{As}_{3}$ is retained for $x \leq 0.06$ [20], the non-magnetic Lu is uniformly distributed, leading to a statistical segmentation of the spin chains. Theoretically, it is expected that the lowfrequency spectral response of the $S=1 / 2$ AF Heisenberg chain becomes gaped by spin segmentation [21] and the static properties fulfill a scaling law though we are not aware of quantitative comparison with experiments.

Below we present experimental zero-field specific heat and simulation results on $\left(\mathrm{Yb}_{1-x} \mathrm{Lu}_{x}\right)_{4} \mathrm{As}_{3}(x=0,0.01$, 0.03). Experimentally, we observe a strong reduction of the low-temperature specific heat. Using the same exchange coupling $J / k_{B}=28 \mathrm{~K}$ as for pure $\mathrm{Yb}_{4} \mathrm{As}_{3}$ [18] and assuming a random distribution of the non-magnetic impurities with concentration $x$ we calculate the average energy gap. The gap depends strongly on the size of the finite segments. Although for small concentrations (of the order of $1 \%$ ) the average gap is small, its impact on the specific heat is strong below $10 \mathrm{~K}$. Our calculation quantitatively explains the reduction of the specific heat and the experimental results confirm the scaling behavior expected for segmented Heisenberg spin chains.

\section{EXPERIMENTAL DETAILS}

The experiments have been performed on single crystals of $\left(\mathrm{Yb}_{1-x} \mathrm{Lu}_{x}\right)_{4} \mathrm{As}_{3}$, characterized previously [20, 22]. Small pieces of mass $9.6 \mathrm{mg}(x=0.01)$ and 2.9 mg $(x=0.03)$ have been investigated in a commercial micro-calorimeter from Oxford Instruments. In the previous paper [18] measurements of the specific heat were performed on the poly-domain sample of the undoped $\mathrm{Yb}_{4} \mathrm{As}_{3}$. The experimental results both for the pure and for the doped system are displayed in Fig. 11 Experimental specific heat $C_{\text {exp }}$ consists of two components: the magnetic part $C_{m a g n}$ and its lattice counterpart $C_{p h}$. The magnetic contribution in zero field for $\mathrm{Yb}_{4} \mathrm{As}_{3}$ was determined theoretically within the Bethe ansatz (BA) solution expressed by the Pade approximants [9]. From the comparison of the total specific heat with the Bethe ansatz estimation of the magnetic heat capacity, we have previously determined the phonon contribution to the heat capacity of $\mathrm{Yb}_{4} \mathrm{As}_{3}$ as

$$
C_{\mathrm{ph}}=\alpha T^{3}+\beta T^{5}
$$

with $\alpha=1.11 \times 10^{-3} \mathrm{~J} /\left(\mathrm{molK}^{4}\right)$ and $\beta=4.9 \times$ $10^{-6} \mathrm{~J} /\left(\mathrm{molK}^{6}\right)$ [18].

In Fig. 1 the BA results are plotted by continuous line and yield the optimal fit to the experimental part $C_{\text {magn }}$ represented by the open circles for the antiferromagnetic coupling $J / k_{B}=28 \mathrm{~K}$. In addition, the full circles show the total specific heat for the pure compound $\mathrm{Yb}_{4} \mathrm{As}_{3}$.

Since we do not expect a change of the phonon component by the small partial substitution of $\mathrm{Yb}$ by $\mathrm{Lu}$,

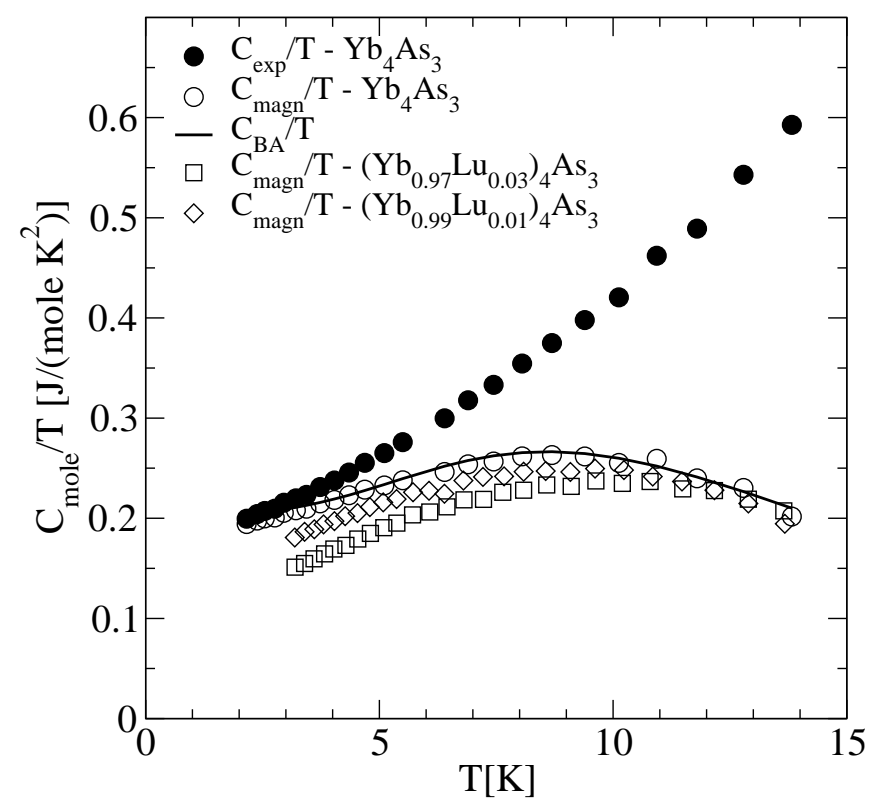

FIG. 1: Experimental results of zero-field specific heat for pure $\mathrm{Yb}_{4} \mathrm{As}_{3}$ system and diluted $\left(\mathrm{Yb}_{1-x} \mathrm{Lu}_{x}\right)_{4} \mathrm{As}_{3}$ for different impurity concentrations $x$.

we have subtracted the same phonon contribution from the data in this study. Note, that we display the data per mol $\left(\mathrm{Yb}_{1-x} \mathrm{Lu}_{x}\right)_{4} \mathrm{As}_{3}$, i.e. the heat capacity has not been rescaled to the amount of magnetic sites in the system. In Fig. 1 the diamonds and squares demonstrate the magnetic part of the specific heat measured for the system with impurities. The observed reduction of $C / T$ with $x$ is far larger than the natural reduction due to the 1 or $3 \%$ decrease of magnetic degrees of freedom for $x=0.01$ and $x=0.03$, respectively.

\section{DESCRIPTION OF THE MODEL AND THE SIMULATION TECHNIQUE}

Computer simulations of the zero-field specific heat of the diluted $\left(\mathrm{Yb}_{1-x} \mathrm{Lu}_{x}\right)_{4} \mathrm{As}_{3}(x=0.01,0.03)$ systems are based on the $S=1 / 2$ isotropic Heisenberg model for each segment consisting of finite number $N$ of spins:

$$
\mathcal{H}=J \sum_{i=1}^{N} \mathbf{S}_{i} \mathbf{S}_{i+1}
$$

The thermodynamic properties of $\left(\mathrm{Yb}_{1-x} \mathrm{Lu}_{x}\right)_{4} \mathrm{As}_{3}$ are described by a fixed value for the exchange coupling $J / k_{B}=28 \mathrm{~K}$ which was found for the pure compound. They are calculated from the derivatives of the free energy related to the partition function $\mathcal{Z}$, using the definition

$$
\mathcal{Z}=\operatorname{Tr} e^{-\beta \mathcal{H}}
$$


The partition function cannot be calculated directly for large $N$ because of non-commuting operators in (11). To eliminate this restriction, we look for systematic approximants to the partition function $\mathcal{Z}$, mapping the quantum system onto a classical one. We express Hamiltonian (1) in terms of the spin-pair operators $\mathcal{H}_{i, i+1}$ as a sum of two noncommuting parts [6]

$$
\begin{aligned}
\mathcal{H}=\mathcal{H}^{\text {odd }}+\mathcal{H}^{\text {even }}= & \left(\mathcal{H}_{1,2}+\ldots+\mathcal{H}_{2 i-1,2 i}+\ldots\right)+ \\
& +\left(\mathcal{H}_{2,3}+\ldots+\mathcal{H}_{2 i, 2 i+1}+\ldots\right)
\end{aligned}
$$

where each part is defined by the commuting components $\mathcal{H}_{i, i+1}$. The series of the classical approximants can be found, using the general Suzuki-Trotter formula [6]. The partition function is calculated as the limit of the corresponding approximants

$$
\begin{array}{r}
\mathcal{Z}=\lim _{m \rightarrow \infty} \mathcal{Z}_{m}= \\
=\lim _{m \rightarrow \infty} \operatorname{Tr}\left[\prod_{i=1}^{N / 2} \mathcal{V}_{2 i-1,2 i} \prod_{i=1}^{N / 2} \mathcal{V}_{2 i, 2 i+1}\right]^{m},
\end{array}
$$

where $\mathcal{V}_{i, i+1}=e^{-\beta \mathcal{H}_{i, i+1} / m}, i=1,2, \cdots, N$ and $m$ is a natural number (reffered to as the Trotter number).

The approximant $\mathcal{Z}_{m}$ can be calculated numerically, without any restrictions on the value of $N$, by the quantum transfer-matrix (QTM) method. The computation of $\mathcal{Z}_{m}$ is possible for relatively small values of $m$, because of computer storage limitation, but for $m$ large enough the leading errors the finite- $m$ approximants are of the order of $1 / m^{2}$ and therefore, extrapolations to $m \rightarrow \infty$ can be performed and the accurate estimates of $\mathcal{Z}$ can be obtained.

To describe properties of the diluted system, we have to calculate the partition function and specific heat for the finite segments of the size $N$. We need to define the two vectors which act in the Hilbert space $\mathcal{H}^{2 m}$ [6]:

$$
\begin{aligned}
& |a\rangle=\sum_{\left\{S^{z}\right\}} \prod_{r=1}^{2 m} \delta_{S_{2 r-1}^{z}, S_{2 r}^{z}}^{z}\left|S_{1}^{z} \ldots S_{2 m}^{z}\right\rangle \\
& |b\rangle=\sum_{\left\{S^{z}\right\}} \prod_{r=1}^{2 m} \delta_{S_{2 r}^{z}, S_{2 r+1}^{z}}^{z}\left|S_{1}^{z} \ldots S_{2 m}^{z}\right\rangle
\end{aligned}
$$

Then the $m$-th classical approximant to the partition function of Eq. (1) is of the form:

$$
\begin{gathered}
\mathcal{Z}_{m}=\left\langle b\left|\left(\mathcal{W}_{1} \mathcal{W}_{2}\right)^{(N-1) / 2}\right| a\right\rangle \text { for odd } N \\
\mathcal{Z}_{m}=\left\langle b\left|\left(\mathcal{W}_{1} \mathcal{W}_{2}\right)^{N / 2}\right| a\right\rangle \text { for even } N
\end{gathered}
$$

where the operators $\mathcal{W}_{1}$ and $\mathcal{W}_{2}$ are defined:

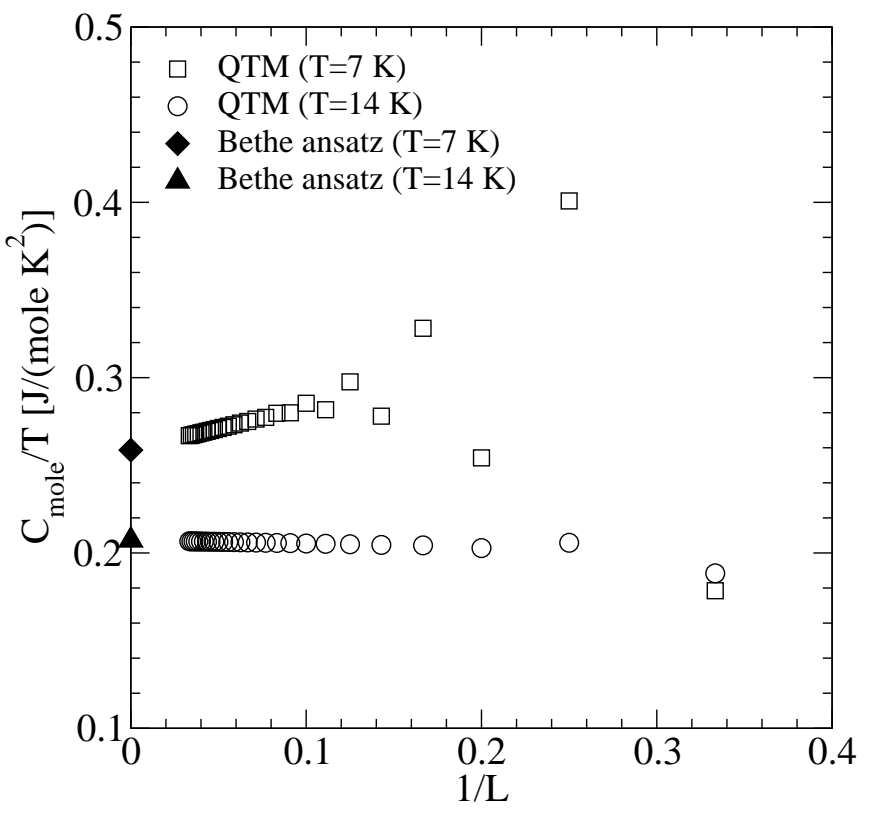

FIG. 2: Size dependence of zero-field specific heat calculated for finite segments with different number of sites $L$. The QTM data are shown by open symbols. The filled symbols represent the Bethe ansatz results corresponding to the macroscopic limit.

$$
\mathcal{W}_{1}=\left(\mathcal{P}^{2} \mathcal{V}_{1,2}\right)^{m}, \mathcal{W}_{2}=\left(\mathcal{P}^{2} \mathcal{V}_{2,3}\right)^{m}
$$

and a unitary shift operator:

$$
\mathcal{P} \equiv \sum_{S_{1}^{z}} \ldots \sum_{S_{2 m}^{z}}\left|S_{2}^{z} S_{3}^{z} \ldots S_{2 m}^{z} S_{1}^{z}\right\rangle\left\langle S_{1}^{z} S_{2}^{z} S_{3}^{z} \ldots S_{2 m}^{z}\right|
$$

For each finite $N$ the specific heat is given as second derivative of the free energy with respect to temperature, where the free energy is given by the formula $\mathcal{F}=-k_{B} T \ln \mathcal{Z}$.

In order to improve the accuracy of the extrapolations the analysis of the specific heat $C_{m}$ as a function of $1 / \mathrm{m}^{2}$ was made using a function described by the extrapolation polynomial of the degree $k\left(k=1, \ldots, k_{\max }\right)$

$$
f\left(1 / m^{2}\right)=a_{0}+\sum_{j=1}^{k} a_{j} \cdot\left(\frac{1}{m^{2}}\right)^{j},
$$

where the extrapolated value of specific heat $C / T=a_{0}$. This extrapolation method was invented in [18] for the infinite spin chains and will be used in this paper to calculate the specific heat for the finite spin chains occurring in the case of the diluted system $\left(\mathrm{Yb}_{1-x} \mathrm{Lu}_{x}\right)_{4} \mathrm{As}_{3}$. 


\section{THE ZERO-FIELD SPECIFIC HEAT OF DILUTED SAMPLE}

To describe the magnetic specific heat of diluted $\left(\mathrm{Yb}_{1-x} \mathrm{Lu}_{x}\right)_{4} \mathrm{As}_{3}$ we need to calculate the contribution $C_{L}$ of a finite chain with $L$ sites. Assuming the uniform distribution of non-magnetic $\mathrm{Lu}$-ions among the chains, each site in the $\mathrm{Yb}^{3+}$-chain is randomly occupied by a magnetic ion with a probability $p=1-x$. The probability of two arbitrary sites being occupied is $p^{2}$. The probability of one end having an empty neighbour is $(1-p)$ and the probability of finding cluster with $L$ sites is $p^{L}(1-p)^{2}$. The total number of $L$-clusters is $n_{L}=N p^{L}(1-p)^{2}(N \rightarrow \infty$ is the total chain length and is much larger than the cluster length). The total number of all $L$-clusters $(L=1, \cdots, \infty)$ is given by the following sum:

$$
\begin{aligned}
n_{t} & =\sum_{L=1}^{\infty} n_{L}=N \sum_{L=1}^{\infty} p^{L}(1-p)^{2}= \\
& =N(1-p)^{2} \sum_{L=1}^{\infty} p^{L}=N(1-p) p .
\end{aligned}
$$

Using the probability distribution of chains with $L$ sites

$$
w_{L}=\frac{n_{L}}{n_{t}}=\frac{N p^{L}(1-p)^{2}}{N p(1-p)}=p^{L-1}(1-p)
$$

we obtain the specific heat per spin [23]:

$$
C=\frac{n}{N} \sum_{L=1}^{\infty} w_{L} C_{L}
$$

where $C_{L}$ denotes the specific heat of a finite chain with $L$ sites, $N$ is the number of all the spins in the system and $n$ is the number of chains (where $n / N=x=(1-p)$ ).

We note that the specific heat per mole of $\left(\mathrm{Yb}_{1-x} \mathrm{Lu}_{x}\right)_{4} \mathrm{As}_{3}$ is given as $(1-x) C$ and finally we have

$$
\begin{array}{r}
C=x \sum_{L=1}^{\infty} p^{L-1}(1-p)(1-x) C_{L}= \\
=x^{2} \sum_{L=1}^{\infty}(1-x)^{L} C_{L},
\end{array}
$$

where $C_{L}=L C_{s p}(L)$ and $C_{s p}(L)$ denotes specific heat per spin. According to the formula (15) the specific heat of the diluted system is determined by all the possible finite size contributions $C_{L}$ which seems unfeasible to achieve. However, for $N$ large enough we enter the asymptotic region where $C_{L}$ varies as $1 / L$ which is demonstrated in Fig. 2

For each temperature we have calculated the specific heat $C_{s p}(L)$ for $L \leq 30$. Our specific heat results for two temperatures $(T=7$ and $T=14 \mathrm{~K})$ are shown in

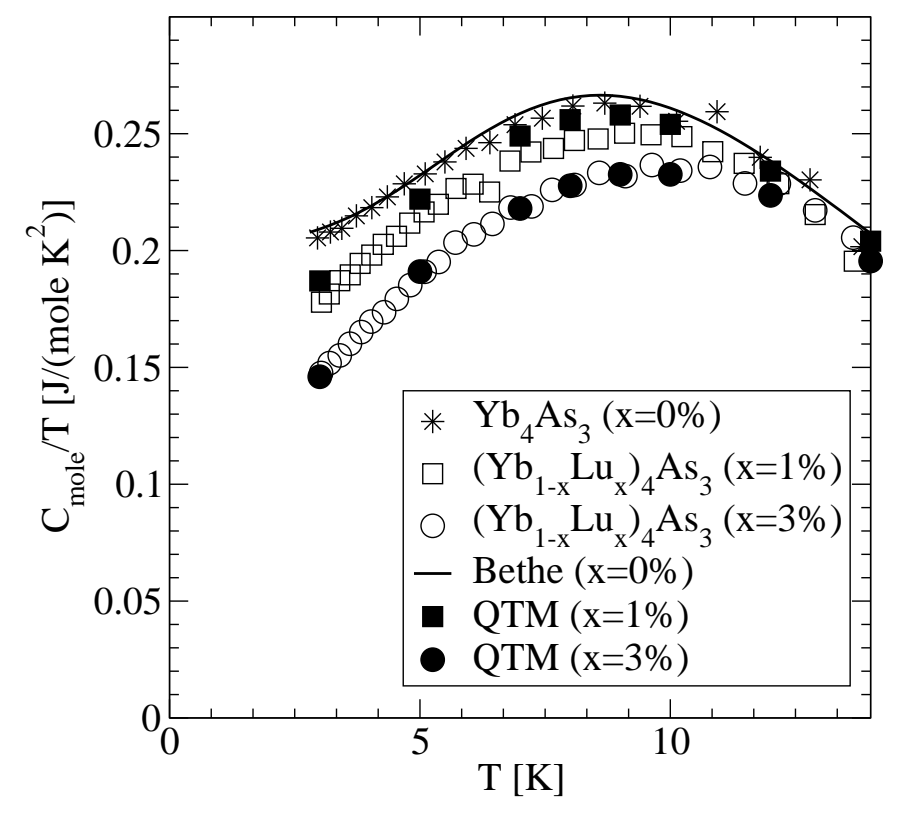

FIG. 3: Temperature dependence for the zero-field specific heat of $\left(\mathrm{Yb}_{1-x} \mathrm{Lu}_{x}\right)_{4} \mathrm{As}_{3}$ for different impurity concentrations $x$. Open symbols represent experimental data for $x \neq 0$ and the corresponding filled symbols indicate the simulation results. The experimental and numerical zero-field data for pure $\mathrm{Yb}_{4} \mathrm{As}_{3}$ are also presented by asterisks and the continuous line, respectively.

Fig. 2. The open symbols represent the specific heat for various numbers of sites $L$. The filled symbols represent zero-field specific heat data $C_{B A}$ for infinite chains, which are exact Bethe ansatz results [9]. For sufficiently large $L>L_{0}$ we can estimate the specific heat by the linear function:

$$
C_{s p}(L)=C_{B A}+a \frac{1}{L}
$$

so that

$$
\begin{aligned}
& C\left(L>L_{0}\right)=x^{2} \sum_{L=L_{0}+1}^{\infty}(1-x)^{L} L\left(C_{B A}+\frac{a}{L}\right)= \\
& =x^{2} C_{B A} \sum_{L=L_{0}+1}^{\infty}(1-x)^{L} L+x^{2} \sum_{L=L_{0}+1}^{\infty} a(1-x)^{L} .
\end{aligned}
$$

Finally, using in Eq. (17) the formula for the geometric series, we have specific heat for $L>L_{0}$ :

$$
\begin{array}{r}
C\left(L>L_{0}\right)=C_{B A}(1-x)^{L_{0}+1}\left(L_{0} x+1\right)+ \\
+a x(1-x)^{L_{0}+1}
\end{array}
$$

and specific heat for whole range of $L$ : 


$$
C=x^{2} \cdot \sum_{L=1}^{L_{0}}(1-x)^{L} \cdot C_{s p}(L) L+C\left(L>L_{0}\right) .
$$

In Fig. 3 we compare experimental data and numerical results obtained from Eq. (19) for the zero-field specific heat of $\left(\mathrm{Yb}_{1-x} \mathrm{Lu}_{x}\right)_{4} \mathrm{As}_{3}(x=0.01$ and $x=0.03)$. A very good quantitative agreement is found in the temperature region analyzed so that the strong reduction of the heat capacity with $x$ is nicely reproduced within our theoretical model.

\section{AVERAGE ENERGY GAP}

Similar calculations can be performed to obtain the average energy gap for a given concentration of impurities. If we define $\Delta_{L}$ as energy gap for the segment with length $L$, the average energy gap is described by the following equation:

$$
\Delta / J=x^{2} \cdot \sum_{L=1}^{\infty}(1-x)^{L} \Delta_{L} / J .
$$

The energy gaps for $L \leq 20$ were calculated within the exact diagonalization technique and are presented as a function of $L$ by the symbols in Fig. 4a. In the case of even number of spins per segment $L$ the ground state is a nonmagnetic singlet, whereas it is a magnetic doublet for odd $L$ [21]. In both cases $\Delta_{L}$ represents the gap from the respective ground to the first excited states.

As expected, the gaps $\Delta_{L}$ decrease with the size $L$ and their asymptotic behavior

$$
\Delta_{L} / J=\frac{\alpha}{L}+\frac{\beta}{L \ln (L)},
$$

is known from the conformal field theory (CFT) approach [21, 24, 25], where $\alpha=\pi^{2} / 2=4.93$ [26] for the the even $L$. We consider also the modified expressions

$$
\frac{L \Delta_{L}}{J}=\alpha+\frac{\beta}{\ln (L)}
$$

and

$$
\ln (L) \frac{L \Delta_{L}}{J}=\alpha \ln (L)+\beta .
$$

In the limit of very long segments the values $\alpha, \beta$ found from Eqs. (21)-(23) should be the same, but for smaller $L$ they may be different and also depend on the number of data points considered. The expressions (21)-(23) are represented in Figs. 4a , 4 $\mathrm{b}$ and 4k, respectively, and are also referred to as the cases I, II, III. In the fits presented, only the lowest values $L=2,3$ were discarded. The corresponding estimates of the parameters $\alpha, \beta$ are quoted in the legend of a given figure. For the even-numbered
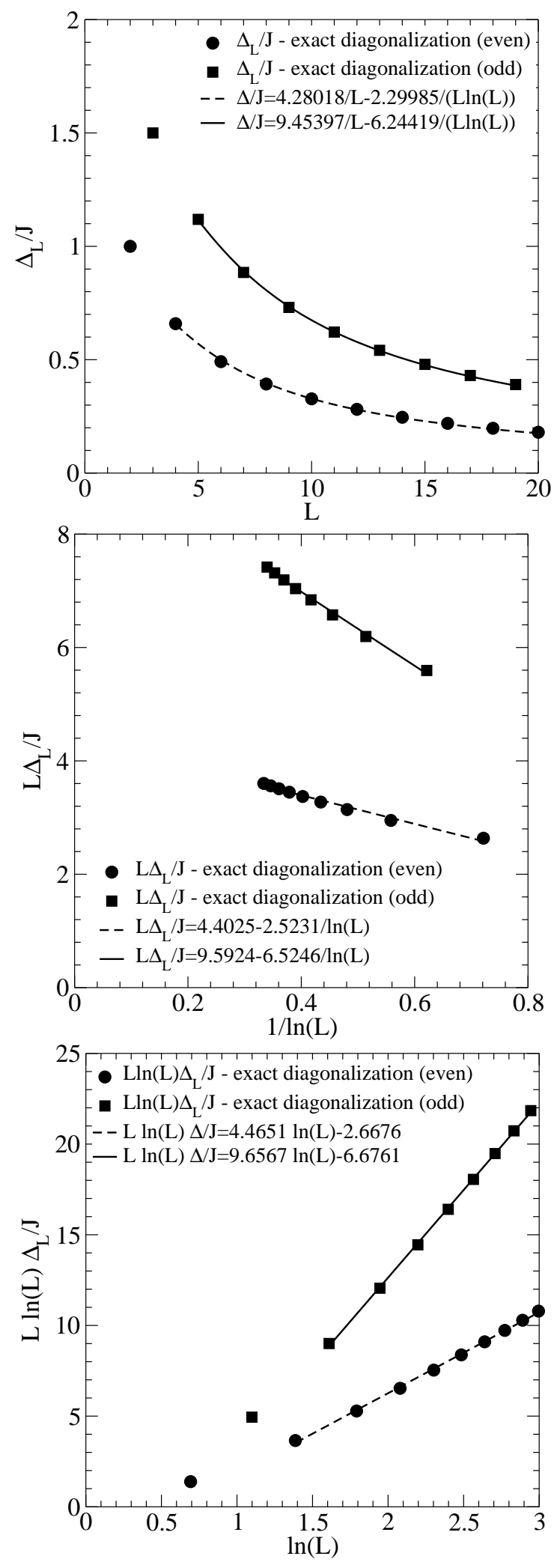

FIG. 4: Size dependence of the energy gaps $(2 \leq L \leq 20)$ for the cases I, II, III. The circles represent estimates for even number of sites $L$ and the line is the result of a fit to the corresponding expression. The infinite system is gapless. 


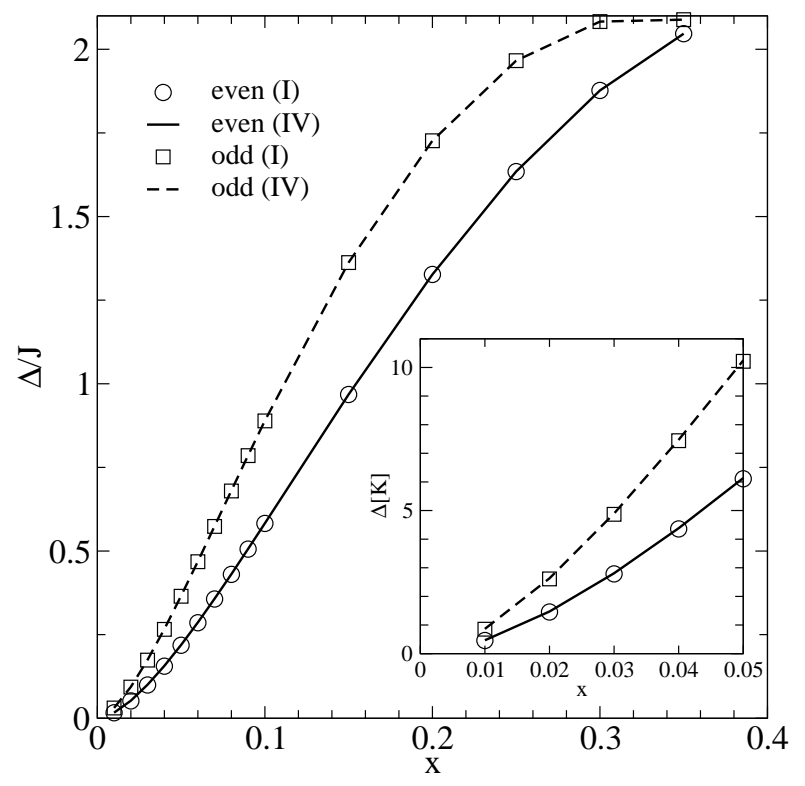

FIG. 5: Dependence of the excitation energy on the concentration of non-magnetic impurities $x$. In the inset the low concentration part of the dependence is enlarged and expressed in the absolute units.

segments $\alpha=4.28,4.40,4.47$ in the case I, II, III, respectively. If the logarithmic correction is neglected (i.e. $\left.\Delta_{L} / J=\frac{\alpha}{L}\right)$ and two data points with the highest $L=$ 18 and 20 are taken into account (the case IV), then $\alpha=$ 4.78, $\beta=-3.52$. All these estimates of $\alpha$ are consistent with the CFT value $\alpha=4.93$.

Having found the coefficients $\alpha$ and $\beta$ determining the asymptotic behavior of $\Delta_{L} / J$, the dependence of the average energy gap on the concentration of impurities $x$ can be estimated from Eq. (20). Excitation energies for the even and odd segments are plotted in Fig 5 for the cases I and IV. For each type of segment, the results are not sensitive to the values $\alpha$ and $\beta$, suggesting that the contribution from the long segments is small. The average energy gap coincides with that calculated for even segments. For small concentrations $x \sim 1-3 \%$ the average gap is small, nevertheless its impact on the specific heat is strong and leads to its substantial reduction.

\section{SCALING BEHAVIOR}

It is known from literature and our previous paper [18] that pure $\mathrm{Yb}_{4} \mathrm{As}_{3}$ is an almost ideal example of a linear Heisenberg antiferromagnet. Here we have also proven that in the diluted system the experimental specific heat data can be described by the same Heisenberg model. This implies that our compound with non-magnetic randomly distributed impurities is a good realization of segmented Heisenberg spin- $1 / 2$ chains.

For these chains the following low-temperature scaling

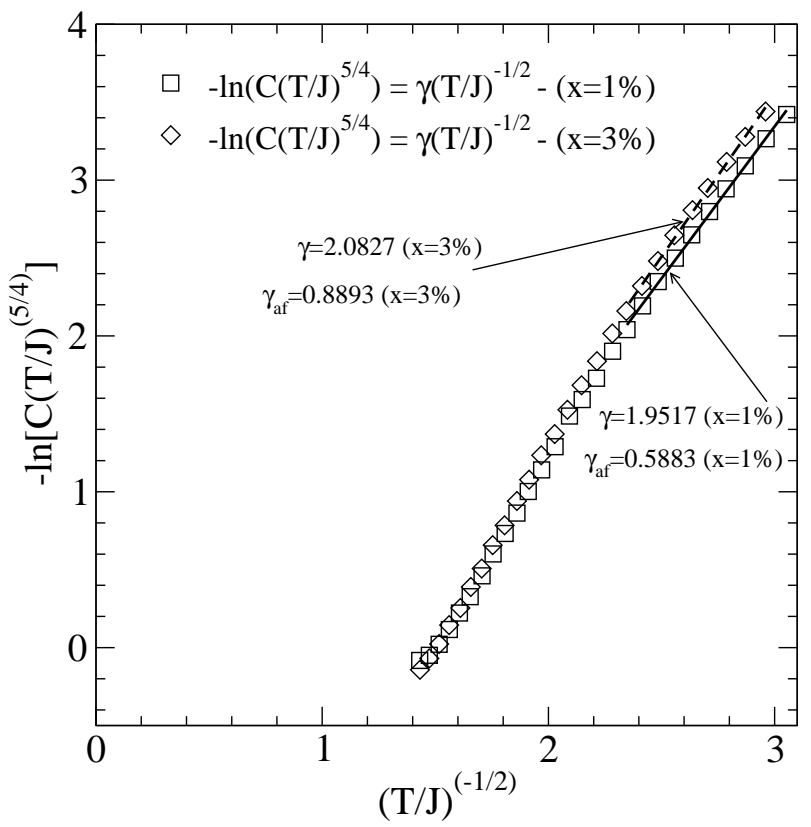

FIG. 6: The scaling behavior of the low temperature specific heat of diluted $\mathrm{Yb}_{4} \mathrm{As}_{3}$ for impurity concentrations $x=1 \%$ and $3 \%$. The linear dependence is observed for the highest values of the scaling variable.

behavior 21] of the specific heat was found in terms of the scaling variable $(T / J)^{-1 / 2}$

$$
\ln \left(C\left(\frac{T}{J}\right)^{5 / 4}\right)=\Phi-\gamma\left(\frac{T}{J}\right)^{-1 / 2}
$$

where $\gamma$ is the $x$-dependent amplitude and $\Phi$ is a constant independent of the scaling variable. The scaling variable depends on the ratio $T / J$ so that the dependence (24) can be verified provided that the magnetic coupling constant is known which in our case amounts to $J / k_{B}=28 \mathrm{~K}$.

In Fig. 6] the experimental results rescaled according to Eq. (24) are plotted as a function of the scaling variable. In the lowest temperature region they display the expected linear dependence. This indicates that our experimental results confirm the scaling behavior of the low-temperature specific heat. This fact is important on its own but it also independently confirms our choice of magnetic coupling constant.

The $\gamma$ values estimated are listed in Fig. 6 and compared with $\gamma_{a f}$ calculated from the analytical formula quoted in literature 21]. They agree qualitatively but do not coincide. We attribute the deviations to the limited validity of the analytical formula which was derived for high concentrations.

We note that the scaling law (24) is fulfilled only for finite $x$. In the limit $x=0$ corresponding to the infinite $\mathrm{S}-1 / 2$ spin chains, the low temperature specific heat is linear in $T$ so that Eq. (24) cannot be satisfied. 


\section{CONCLUSIONS}

We have proven that the spin model worked out for the pure compound $\mathrm{Yb}_{4} \mathrm{As}_{3}$ can also explain the specific heat data in the presence of non-magnetic impurities, assuming their random distribution. We have applied the quantum transfer matrix method to obtain the accurate numerical results without any adjustable parameter.

This conclusion implies that the diluted compound $\left(\mathrm{Yb}_{1-x} \mathrm{Lu}_{x}\right)_{4} \mathrm{As}_{3}$ studied here is a good realization of segmented antiferromagnetic Heisenberg spin-1/2 chains with frozen impurities and the low temperature specific heat data can be used to verify experimentally the theoretically expected scaling law. We have demonstrated that the lowest temperature tail of the logarithm of the rescaled specific heat as a function of $(T / J)^{-1 / 2}$ (Eq. (24) ) fulfills the linear dependence predicted by conformal field theory. This finding also provides indepen- dent evidence for the correct choice of the magnetic coupling constant which is unchanged compared to the pure system. We have also confirmed that the amplitude of the leading term determining the energy gap as a function of the size $L$ for even segments is consistent with the earlier theoretical prediction. The impurity driven reduction of the specific heat can be attributed to the finite energy gaps appearing in the even-numbered segments.

\section{Acknowledgments}

P.G. likes to acknowledge discussions with Andreas Honecker. This work was supported by the Polish Ministry of Science and High Education grant N202 230137 and was granted access to the HPC resources in PSNC Poznań (Poland) and those available within DECI program by the PRACE-2IP under grant no RI-283493
[1] F. D. M. Haldane, Phys. Rev. Lett. 50 (1983) 1153.

[2] P. Renard, M. Verdagner, L. P. Regnault, W. A. C. Erkelens, J. Rossat-Mignat, J. Ribas, W. G. Stirling, C. Vettier, J. Appl. Phys. 63 (1988) 3538.

[3] A. Caramico D'Auria, U. Esposito, F. Esposito, D. Gatteschi, G. Kamieniarz, S. Wałcerz, J. Chem. Phys. 109 (1998) 1613.

[4] G. Kamieniarz, R. Matysiak, A. Caramico D'Auria, F. Esposito, U. Esposito, Phys. Rev. B 56 (1997) 647

[5] J. C. Bonner, M. E.Fisher, Phys. Rev. A135 (1964) 640.

[6] T. Delica, H. Leschke, Physica A176 (1990) 736.

[7] G. Kamieniarz, M. Bieliński, J.-P. Renard, Phys Rev. B 60 (1999) 14521.

[8] A. Caramico D'Auria, U. Esposito, F. Esposito, G. Kamieniarz, R. Matysiak, J. Phys.: Condens. Matter 13 (2001), 2017.

[9] D. C. Johnston, R. K. Kremer, M. Troyer, X. Wang, A. Klümper, S. L. Budko, A. F. Panchula, P. C. Canfield, Phys. Rev. B 61 (2000) 9558.

[10] N. Shibata, K. Ueda, J. Phys. Soc. Jpn. 70 (2001) 3690.

[11] G. Kamieniarz, R. Matysiak, A. Caramico D'Auria, F. Esposito, C. Benelli, Computer Physics Communications 147 (2002) 194.

[12] G. Kamieniarz, R. Matysiak, Computational Materials Science 28 (2003) 353.

[13] M. Antkowiak, P. Kozłowski, G. Kamieniarz, G.A.
Timco, F. Tuna, P.E.P. Winpenny, Phys. Rev. B, in press [14] M. Oshikawa, I. Affleck, Phys. Rev. Lett. 79 (1997) 2883.

[15] B. Schmidt, H. Aoki, T. Cichorek, J. Custers, P. Gegenwart, M. Kohgi, M. Lang, C. Langhammer, A. Ochiai, S. Paschen, F. Steglich, T. Suzuki, P. Thalmeier, B. Wand, and A. Yaresko, Physica B 300 (2001) 121.

[16] M. Kohgi, K. Iwasa, J.-M. Mignot, A. Ochiai and T. Suzuki, Phys. Rev. B 56 (1997) R11388.

[17] M. Kohgi, K. Iwasa, J.-M. Mignot, B. Fak, P. Gegenwart, M. Lang, A. Ochiai, H. Aoki, T. Suzuki, Phys. Rev. Lett. 86 (2001) 2439.

[18] R. Matysiak, G. Kamieniarz, P. Gegenwart, A. Ochiai, Phys. Rev. B 79 (2009) 224413.

[19] R. Matysiak, Acta Physica Polonica A 118 (2010) 969.

[20] H. Aoki, Ph.D. thesis, Tohoku University (2000), unpublished.

[21] S. Wessel and S. Haas, Phys. Rev. B 61 (2000) 15262.

[22] A. Ochiai, H. Aoki, T. Suzuku, JJAP Series 11 (1999) 117.

[23] H. Asakawa, M. Matsuda, K. Minami, H. Yamazaki, K. Katsumata, Phys. Rev. B 57 (1998) 8285.

[24] I. Affleck, S. Qin, J. Phys. A: Math. Gen. 32 (1999) 7815.

[25] S. Haas, Phys. Rev. Lett. 80 (1998) 4052.

[26] J. L. Cardy, J. Phys. A: Math. Gen. 19 (1986) L1093. 